\title{
Functional significance of the affiliative smile
}

\author{
JOAN S. LOCKARD, RENATE I. MCVITTIE, and LISA M. ISAAC \\ University of Washington, Seattle, Washington 98195
}

\begin{abstract}
Common human social signals, such as smiling, are often attributed certain functions without documentation. The intent of this study was to ascertain the efficacy of the affiliative smile in a minimal social space. Elevators were chosen as the observational setting, since dyadic interactions have a definite beginning (entrance) and ending (exit). Eight hundred adults (males and females, ages 19-60 years) were observed as they entered individually an elevator where a male or female observer stood in one of nine equally divided floor cells and displayed either an affiliative smile or a neutral face (no smile). In the affiliative situation (particularly for female observers), the distance between the subject and the observer was significantly shorter than in the neutral situation, where the distance was maximized.
\end{abstract}

Common human social signals, such as smiling, are often attributed certain functions without documentation. In friendly exchanges, smiling between individuals is a frequent occurrence. Under such circumstances a smile is indicative of the intent or mood of the situation. However, whether it can be employed to increase affiliative behavior in "neutral" to somewhat threatening settings has not be quantified under well-controlled conditions.

The present research attempted to ascertain the affiliative efficacy of smiling in a minimal social space. Elevators were chosen as the observational environment because of their size and atmosphere of enclosure, and also since interactions in this setting have a definite beginning (entrance of an individual) and ending (exit by that individual). Two studies were conducted: first, preliminary observations $(\mathrm{N}=88)$ to determine if persons maximize interpersonal distance in elevators and are in fact in a minimal social space; and a second, more extensive study $(\mathrm{N}=800)$ to test if smiling functions as an affiliative signal to decrease that distance.

Implicit in many of the studies in the literature on social spacing is the hypothesis that there exists for any given situation an optimal spatial arrangement (Hall, 1964; Sommer, 1959). It is proposed that this arrangement is dependent on the relationship between the individuals and on the setting (Argyle \& Dean, 1965). With some data as support, the underlying assumption in these studies is that the extent of involvement of two relatively distant individuals may be increased by increasing eye contact (e.g., Exline, Gray, \& Schuette, 1965), gestures (e.g., Ekman \& Friesen, 1967; Mehrabian, 1968), and/or body orientation (e.g., Pedersen \& Heaston, 1972). By utilizing minimal social spacing, the

The authors wish to thank W. Bernds and H. Klein for their assistance in Study 1; J. Gaston, A. Hartley, J. Sevenich, C. Peck, S. Takano, and M.Smith, who were instrumental in the conduct of Study 2; and S. Franklin and B. Trent for their help in data collation, analyses, and graphics. present study is a critical test of that assumption with respect to smiling and its supposedly affiliative function.

\section{METHODS AND RESULTS}

\section{Study 1}

Three student observers (two males and one female) and three elevators (varying in area from 37 to $48 \mathrm{sq} \mathrm{ft}$ ) at the University of Washington, Seattle, were employed. The floor area of an elevator was divided into nine equal "imaginary" spaces containing the same number of floor tiles (Figure 1). An observer positioned him. self randomly in one of these boxes and recorded the position of the second individual to enter the elevator (doing so after that subject had exited). The positions of subsequent individuals who entered the elevator were not recorded if the observer was not alone. The observer avoided verbal and visual contact with the subjects. The 88 subjects (both males and females) so recorded ranged in approximate age from 19 to 60 years.

For each position of the observer, the total number of individuals for the three farthest boxes was tabulated. For example, in Figure 1 when the observer was in Position 1, the number of subjects choosing to stand in Boxes 6,8 , and 9 was added and divided by the total number of subjects for that observer's position. The case where the observer positioned himself in Box 2 was not tested, as its location was directly in front of the elevator doors.

At random it would be expected that each box is equally likely to be chosen, so that for any given situation there would be three of eight in the farthest boxes (i.e., for a binomial distribution, $\mathrm{p}=.375, \mathrm{q}=.625$ ). (Box 5 data were not used in the statistical analyses since there were four farthest boxes.) As shown in Figure 2 for each box, the results revealed that 63 of 88 subjects selected one of the three farthest boxes from the observer. Chi square was $43.6, p<.001$ or $z=6.606, p<.0001$.

It is interesting to note that at no time did a subject 


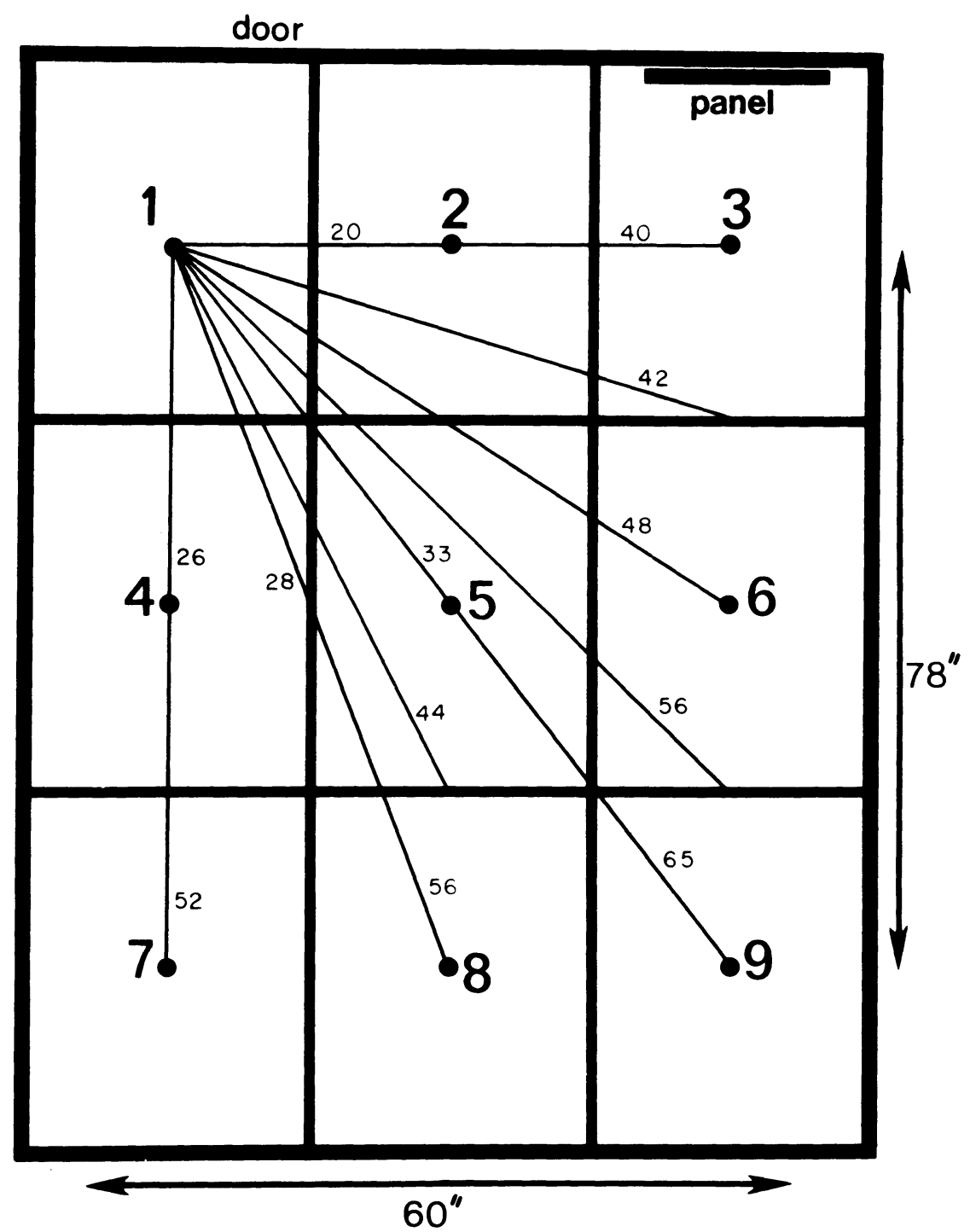

Figure 1. Elevator floor plan: nine equal area boxes and distances (inches) between their center points and boundaries.

position himself in Box 2 in front of the doors. Also, when the observer was in Box 5, eight of nine subjects positioned themselves on the same side as the control panel, whereas, for the other positions of the observer, the location of the panel after the floor button was pushed did not appear to be significant.

\section{Study 2}

Similar to the above procedure, eight observers (five females and three males) used five elevators of equal floor area ( $60 \times 78 \mathrm{in}$.) to record the floor position (one of nine equal boxes or halfway between boxes) selected by 800 college students ( 454 females and 346 males). On half of the trials, the observer smiled and made direct eye contact with the subject entering the elevator, and on half of the trials the observer did neither (baseline condition). The data were collated such that the distance from the center point of each box (or midway between boxes) was determined, as illustrated in Figure 3, and then the approximate distance in inches of the subject relative to the observer was computed.

With each observer as his own control (one half smile and one-half baseline trials) and with the data collapsed across sex of subject, the baseline distance between subject and observer was significantly longer than under the smile condition $(t=5.52, \mathrm{p}<.001)$, as shown in Figure 4 for each box. This result was especially strong for female subjects $(t=5.04, p<.005)$ but was true to a lesser extent for male subjects $(\mathrm{t}=2.52$, $\mathrm{p}<.05)$. For both the smile and baseline condition, the distance between subject (s) and observer (o), when analyzed by sex (M or F) of either, diminished in the 


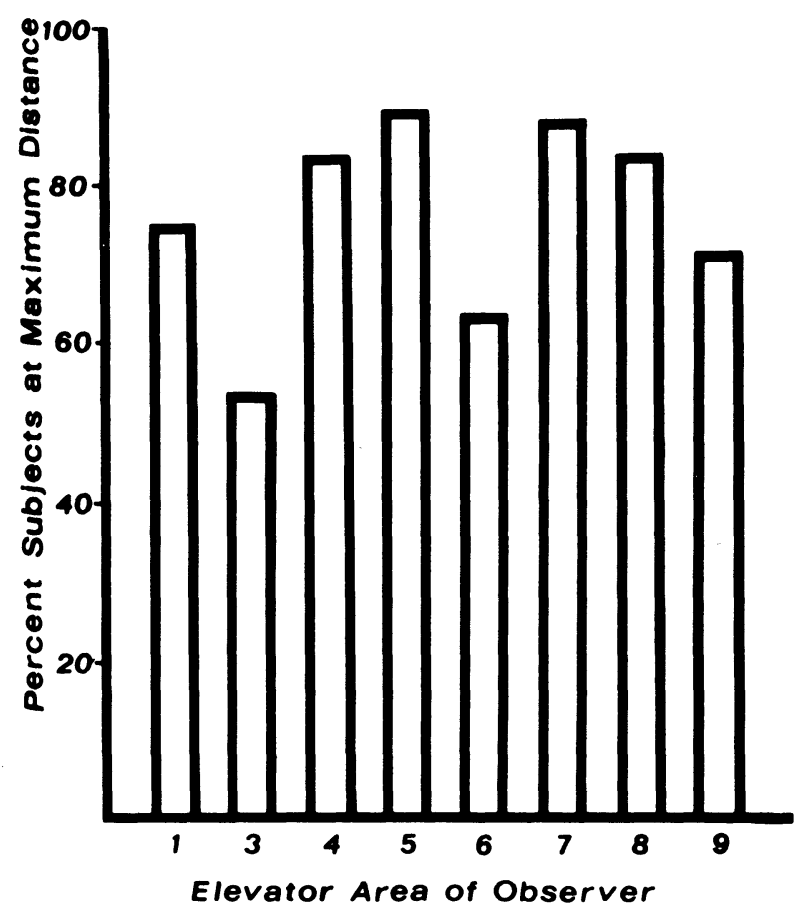

Figure 2. Percentage of subjects at maximum distance from observer: elevator area numbers refer to floor plan of Figure 1.

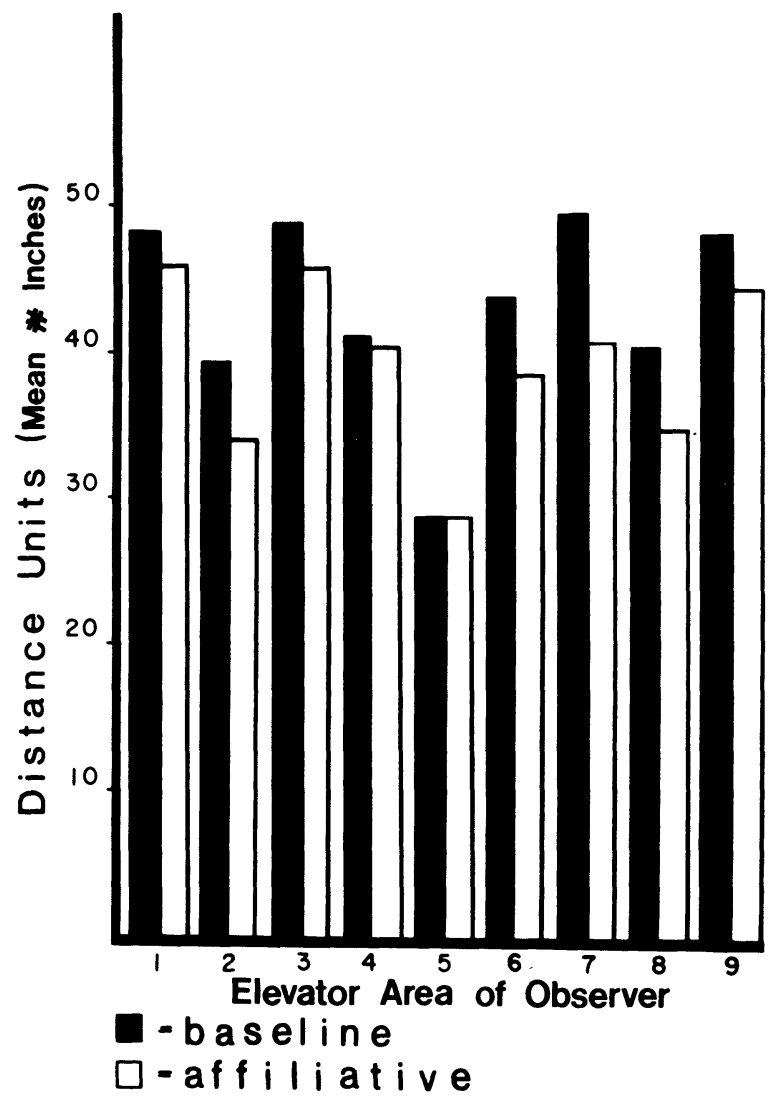

Figure 3. Mean number of inches from observer during baseline and affiliative condition: elevator area numbers refer to floor plan of Figure 1. following order: the greatest distance between $\mathrm{M}_{\mathrm{s}}-\mathrm{M}_{\mathrm{o}}$, next $M_{\mathrm{s}}-\mathrm{F}_{\mathrm{o}}$ and $\mathrm{F}_{\mathrm{s}}-\mathrm{M}_{\mathrm{o}}$, with the shortest distance between $\mathrm{F}_{\mathrm{s}}-\mathrm{F}_{\mathrm{o}}$. These data indicate that females tend to tolerate, in this situation, closer interpersonal distances to one another than any other sex combination (e.g., $\mathrm{t}=2.62, \mathrm{p}<.05$ between $\mathrm{M}_{\mathrm{s}}-\mathrm{F}_{\mathrm{o}}$ and $\left.\mathrm{F}_{\mathrm{s}}-\mathrm{F}_{\mathrm{o}}\right)$.

\section{DISCUSSION}

The findings of these two studies suggest that smiling can function to increase affiliative behavior (namely, proximity) even in what may be potentially a threatening situation (i.e., minimal social spacing), particularly if males are involved. In this sense, the human smile may be analogous to the submissive (fear) grimace of Old World nonhuman primates (e.g., van Hooff, 1971), communicating "no harm intended" to potentially dangerous conspecifics (i.e., dominant animals). Some support for this notion was indicated by the failure of an attempted third elevator study by the present authors. The objective was to displace (supplant) individuals by intruding into their personal space. Male observers either refused immediately or after a single trial of moving one box closer to a subject. Female observers were very "uncomfortable" about participating, with the total outcome being too few observations for statistical analysis.

An overview of the findings of social spacing in the literature which may be relevant to the present research suggests the following consistent patterns: Females choose positions that maintain less interpersonal space from others and they allow people to approach them closer at the sides than frontally (Dosey \& Meisels, 1969). Generally, males will approach females closer than other males and will allow females to approach them closer than other males (Horowitz, Duff, \& Stratton, 1964). Both males and females maintain twice as much distance between themselves and a tall person than between themselves and a short person; the latter is most apparent in the standing position, but also significant in the sitting position (Hartnett, Bailey, \& Hartley, 1974). More easily threatened females are less likely to invade someone's personal space than are males (Felipe \& Sommer, 1966). Also, stress tends to increase the social spacing of both females and males (Dosey \& Meisels, 1969), possibly expanding the buffer zone (Horowitz et al., 1964) which serves as protection against perceived threats.

The data (and lack of data with respect to the intrusion study) of the present research are consistent with the general findings by other investigators and document the affiliative function of human smiling even under less than optimal social conditions, that is, minimal social spacing.

\section{REFERENCES}

ArgYle, M., \& Dean, J. Eye contact, distance and affiliation. Sociometry, 1965, 28, 389-404.

Dosey, M. A., \& Meisels, M. Personal space and self-protection. Journal of Personality and Social Psychology, 1969, 11, 93-97.

Ekman, P., \& Friesen, W. V. Head and body cues in the judgement of emotions: A reformulation. Perceptual and Motor Skills, 1967, 24, 711-724.

Exline, R. V., Gray, D., \& Schuette, D. Visual behavior in a dyad as affected by interview content and sex of respondent. Journal of Personality and Social Psychology, 1965, 1, 201-209.

Felipe, N. J., \& Sommer, R. Invasions of personal space. Social Problems, 1966, 14, 206-214.

HALL, E. T. Silent assumptions in social communication. Disorders of Communication, 1964, 42, 41-55.

Hartnett, J. J., Bailey, K. G., \& Hartley, C. S. Body height, position, and sex as determinants of personal space. Journal of Psychology, 1974, 87, 129-136. 
Horowitz, M. J., Duff, D. F., \& Stratton, L. O. Body buffer zone. Archives General Psychiatry, 1964, 11, 651-656.

Merrabian, A. Relationship of attitude to seated posture, orientation, and distance. Journal of Personality and Social Psychology, 1968, 10, 26-30.

Pedersen, D. M., \& Heaston, A. B. The effects of sex of subject, sex of approaching person, and angle of approach upon personal space. Journal of Psychology, 1972, 82, 277-286.
Sommer, R. Studies in personal space. Sociometry, 1959 , 22, 247-260.

VAN Hooff, J. A. R. A. M. Aspects of the social behaviour and communication in human and higher non-human primates. Rotterdam: Brondar-Offset m.v., 1971.

(Received for publication December 23, 1976.) 